\title{
La banca y las crisis financieras en la literatura popular: Una fortuna peligrosa, de Ken Follett
}

\author{
CARLOS RODRÍGUEZ BRAUN \\ Universidad Complutense de Madrid, Facultad CC.EE., Campus de Somosaguas, 28223 Pozuelo de \\ Alarcón (Madrid), España.E-mail: crb@ccee.ucm.es
}

\begin{abstract}
RESUMEN
El best-seller de Ken Follett, Una fortuna peligrosa, además de los ingredientes habituales del género, contiene abundante información económica, al relatar la historia de un banco ficticio, pero cuyas operaciones y problemas son idénticos a los de los bancos reales, en una sucesión de crisis financieras que efectivamente se registraron en el siglo XIX, en particular las de 1866, 1878 y 1890 en Gran Bretaña, asociadas respectivamente a las quiebras de Overend, Gurney \& Co., el City of Glasgow Bank y la banca Baring Brothers. El contexto histórico, la importancia de los marcos institucionales para el desarrollo económico, y la organización de la banca y el sistema financiero en tiempos en los que empezaba a plasmarse el papel del banco central como prestamista de última instancia, son analizados en general con acierto. La novela, de este modo, traza un retrato que integra abundantes referencias económicas y financieras de interés para economistas e historiadores.
\end{abstract}

Palabras clave: Banca, crisis, quiebras, historia económica, instituciones, cultura.

\section{Banking and Financial Crises in Popular Literature: $A$ Dangerous Fortune, by Ken Follett}

\begin{abstract}
Ken Follett's best-seller, A Dangerous Fortune, in addition to the usual ingredients of this kind of popular literature, contains much economic information: it tells the story of a bank, a fictitious one, but with operations and problems identical to the real ones, in a succession of economic crises that in fact took place in the nineteenth century, particularly those of 1866, 1878 and 1890 in Britain, respectively associated with the bankruptcies of Overend, Gurney \& Co., the City of Glasgow Bank and Baring Brothers bank. The historical context, the relevance of institutional frameworks for economic development, and the organization of banks and the financial system in the times when the central bank role as lender of last resort was barely beginning to take form, are analyzed correctly in general lines. The novel thus draws a picture that includes many economic and financial references that may interest economists and historians.
\end{abstract}

Keywords: Banking, Crises, Bankruptcy, Economic History, Institutions, Culture.

Clasificación JEL: N23, N21, G21, G33, O43

* El autor agradece los comentarios y las sugerencias de los dos evaluadores anónimos.

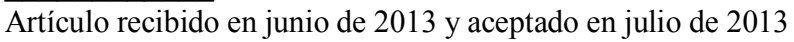

Artículo disponible en versión electrónica en la página www.revista-eea.net, ref. ə-32105

ISSN 1697-5731 (online) - ISSN 1133-3197 (print) 


\section{INTRODUCCIÓN}

La banca ha atraído siempre la atención de la literatura, incluida la más popular. ${ }^{1}$ Ken Follett publicó en 1993 una novela que, como otras suyas, se convirtió en un gran éxito: Una fortuna peligrosa. Él mismo confiesa que se inspiró en la crisis del Banco Baring (Follett, s.d.). Por supuesto, es difícil que un libro sea un best-seller si sólo habla de banca. Una fortuna peligrosa tiene los ingredientes característicos del género, desde el crimen, la codicia y la intriga hasta la ambición, el amor y los celos. Lo inesperado e interesante para el economista, sin embargo, no son esos ingredientes.

Hijo de un inspector de Hacienda, Ken Follett, nacido en Cardiff en 1949, estudió filosofía en el University College de Londres y trabajó unos años como periodista. En 1978 escribió un guión para la BBC sobre Fringe Banking (Ramet 1999, 66), y ese mismo año publicó junto a René L. Maurice The Bank Heist of the Century, revisado y publicado posteriormente con el título Under the Streets of Nice (Maurice y Follet 1989), sobre el robo perpetrado en el banco Société Générale en Niza en 1976. Su estilo ágil está posiblemente vinculado con su experiencia como periodista, y también se observa en otro de sus primeros trabajos de ficción con contenido económico, Paper Money (Follett 1987), que publicó originalmente en 1977 con el seudónimo de Zachary Stone (Ramet 2010,80 ), y que también trata de un robo, aunque esta vez no real: la víctima es el Banco de Inglaterra.

Estos antecedentes sugieren que Follett está familiarizado con el mundo de la economía, y en concreto con el de la banca, lo que resulta diáfano en Una fortuna peligrosa, obra diferente en varios aspectos: es bastante posterior a las que acabamos de mencionar, es más relevante y extensa, y contiene abundantes referencias a la operativa y la historia de la banca y sus marcos institucionales, expresadas la mayoría de las veces con acierto. El énfasis en la banca y sus avatares es tan patente que la trama del libro se corresponde con una sucesión de crisis financieras y económicas que realmente se registraron en el siglo XIX.

\section{LAS CRISIS DE 1866 Y 1873}

Aparecen los protagonistas en el prólogo, en 1866, en primer lugar el héroe, Hugh Pilaster, su primo Edward, la madre de éste, la tía Augusta, y Micky Miranda, nativo del ficticio país latinoamericano "Córdoba". Pusilánime el segundo, los otros tres son ambiciosos, con la diferencia de que el primero tiene escrúpulos y los dos últimos, los grandes villanos, carecen de ellos. Muy al comienzo sabemos que los Pilaster son banqueros, y aparece un hecho económico

\footnotetext{
${ }^{1}$ Sobre crédito y banca en la literatura culta o clásica pueden verse, por ejemplo, los ensayos sobre Shakespeare y Balzac en Perdices de Blas y Santos Redondo 2006. Y también el análisis de El banquero anarquista de Pessoa en Linde 2004.
} 
vinculado con la banca que atravesará toda la novela: las quiebras y su impacto.

Un trabajador, el señor Robinson, vuelve a casa sin cobrar: su jefe, Tobías Pilaster, el padre de Hugh, ha quebrado, y ante la imposibilidad de pagar sus deudas se ha suicidado; se verá que fue víctima inocente de una externalidad negativa: una crisis bancaria. Los dos niños de Robinson, Danny y Maisie, para no ser una carga para sus padres en esas circunstancias tan duras, huyen, y queda planteada la posibilidad de que las personas puedan con su esfuerzo superar las adversidades de la vida; los ricos, como Hugh, y también los pobres, como Danny y Maisie -él emigra a Estados Unidos y ella se abre camino en Inglaterra.

Nos enteramos de las aventuras de los protagonistas, y de la crisis de 1866, en la primera parte de la novela, fechada en 1873.

Antes de conocer prácticamente nada de la familia Miranda, leemos que el padre de Micky, "Papá" Miranda, viene a Londres a comprar rifles. El contraste institucional entre Gran Bretaña y América Latina se repite a menudo en el libro, empezando por la conocida tesis de la herencia colonial, que deja una suerte de feudalismo donde unos caudillos se suceden a otros en un ambiente de inseguridad e inestabilidad. Así, en Córdoba, un país que podría ser México, pero que en realidad es un símbolo de la América Latina decimonónica, la economía no es competitiva sino un constante juego de suma cero presidido por la violencia: desde esos rifles, que Miranda quiere para atacar a una familia rival y robar su valioso nitrato, hasta el empleo de la fuerza para tomar el poder y aprovecharse de él, pasando por varios crímenes cometidos sin miramiento alguno. Los Miranda abordan los negocios con una frase que alude al asesinato, y que evoca la de "una oferta que no pudo rechazar" de El Padrino: “¿quién se interpone en nuestro camino?" (Follett 2011, 85).

En el país que vende las armas, Gran Bretaña, se nos informa de que la división del trabajo y la maquinaria han aumentado considerablemente la productividad de la industria armamentística. Se menciona incluso la fabricación de alfileres, y hay otra alusión smithiana: los viajes de los jóvenes estudiantes por Europa, e incluso la inutilidad de la Universidad de Oxford, aunque no en los profesores sino en los alumnos; en efecto, allí Micky y Edward se dedicaron "a jugar, a beber y a montar sonadas juergas, sin apenas molestarse en fingir que estudiaban" (Follett 2011, 53). ${ }^{2}$

La economía aparece con detalle cuando Micky ilustra a su padre sobre las

\footnotetext{
${ }^{2}$ En La riqueza de las naciones dice Smith: "En la Universidad Oxford la mayoría de los profesores oficiales hace mucho que han renunciado incluso a simular que enseñan" (Smith 2007, 704). En inglés la similitud de las palabras es más marcada; el novelista habla así de los estudiantes de Oxford: "making only the barest pretence of being students" (Follett 1994, 36), mientras que para Smith los profesores de esa Universidad "have...given up altogether even the pretence of teaching" (Smith 1981, vol. 2, 761).
} 
transacciones internacionales y la utilidad de los "bancos mercantiles" (Follett 2011, 52). La traducción de merchant banks en el original inglés (Follett 1994, 35 ) es dudosa, aunque al menos no dice "bancos comerciales", que era algo que el Banco Pilaster claramente no era: no era un banco de depósitos y préstamos personales y comerciales. Banco de inversión o banco de negocios habría sido una traducción más apropiada para referirse a la institución bancaria europea de los merchant banks, ejemplificada en el siglo XIX por dos apellidos: Baring y Rothschild. El nombre "banco de aceptaciones" también habría sido adecuado, porque dichas empresas participaban activamente en tales negociaciones (Flandreu y Ugolini 2011, 6-9). Aportaban liquidez al mercado, en especial de financiación exterior, aceptando letras que después eran descontadas: la clave era, por supuesto, asegurarse la garantía del comprador, o el emisor de los bonos, de que iba a pagar la mercancía en cuestión, o abonar los intereses de los títulos que el banco aceptaba. Se dedicaban a financiar obras públicas internacionales, y, como en el caso de Baring en la Argentina o el Pilaster en Córdoba, con graves riesgos -aunque no aportaban capital o financiación en transacciones de elevado apalancamiento, como sucedió más adelante: lo hemos visto en EE UU y otros países en años recientes (Beauman 1992).

La financiación bancaria resuelve la dificultad de que Papá Miranda quiere pagar los rifles cuando lleguen a su país, mientras que el fabricante quiere cobrarlos antes de que salgan de la fábrica. Micky explica a su padre que el banco paga al vendedor, y cuando lleguen los rifles a Córdoba el dinero no tiene por qué moverse porque se empleará para pagar las exportaciones cordobesas de carne a Inglaterra; explica las comisiones y más adelante los seguros del transporte marítimo.

En contraste con este cacique latinoamericano, aparecen otros empresarios importantes en la trama: los banqueros Greenbourne, judíos ricos, buenos negociantes, y gente honrada. Está el mandamás, Ben, y su hijo Solly, bella persona que "no cursó estudios universitarios ni hizo viaje alguno por Europa, sino que pasó directamente a colaborar en el negocio paterno" (Follett 2011, 56). ${ }^{3}$ Nuevamente aparece la cuestión de las bancarrotas y el problema del contagio en Europa, porque se ha desatado una crisis en Austria.

Otro contraste es entre los hombres de negocio y la aristocracia, mal considerada por los personajes mejores de la novela, como Seth Pilaster, cuyo desdén hacia la nobleza ociosa es tan intenso como su olfato para los negocios: Micky le presenta a su padre, que tiene un rancho, y Seth sin mediar palabra le recomienda que invierta en la actividad frigorífica. Estos banqueros británicos no sólo tienen instinto empresarial sino que además tienen principios, y enlazan la religión con su trabajo. Cuando los Miranda le cuentan a Edward Pilaster que

\footnotetext{
${ }^{3}$ Para la crítica de Adam Smith a esos viajes véase Smith 2007, 712-3.
} 
necesitan financiar la importación de rifles, éste les informa que la operación será vetada por Seth:

Es metodista, ya sabes. Bueno, lo es toda la familia, pero él es más devoto que nadie. De cualquier modo, no financiará ninguna compra de armas, y como es el presidente del consejo ${ }^{4}$,ésa es la política del banco. (Follett 2011, 60)

Mientras que la banca es habitualmente asociada con el materialismo, aquí las dos familias banqueras sobresalientes, los Pilaster y los Greenbourne, son creyentes.

La laboriosidad y la modestia caracteriza a Hugh en el banco: es un pariente pobre, que debe probar sus méritos desde abajo. No es culpable de nada pero su padre se ha arruinado, aunque tampoco por su culpa, y esto tiene relación con el papel de la banca. De todas maneras, Hugh ha de demostrar él mismo su valía, en una familia que es al respecto muy rigurosa: "si uno empezaba a aliviar las penalidades fruto de los fracasos, entonces los frustrados carecerían de incentivo para hacer las cosas como era debido" (Follett 2011, 64). Ese rigor, que lleva a la familia a excluir todo criterio que no sea profesional para dirigir el banco, es precisamente lo que, gracias a las intrigas de Augusta, se va a perder, $\mathrm{y}$, como atestigua la experiencia de tantas empresas familiares, abocará a los Pilaster, una familia comparable a los Rothschild, a una grave crisis. Las maquinaciones de Augusta revelan, además de la radical inmoralidad del personaje, los riesgos de la intervención no profesional en la vida de una empresa familiar: no sólo conspira contra el nombramiento del mejor gestor (Hugh) sino también para que se nombre al peor (Edward), y avala la pésima gestión de éste.

Tobías Pilaster fue víctima de una crisis, de la que ahora se habla:

Cayó el 11 de mayo de 1866, fecha que los banqueros conocían como el Viernes Negro. Aquel día, una firma intermediaria de efectos mercantiles llamada Overend y Gurney, S.L., se declaró en quiebra por cinco millones de libras esterlinas y arrastró en su hundimiento a muchas empresas, entre las que figuraban la Sociedad Bancaria de Londres, la firma constructora de sir Samuel Peto y la razón social Tobias Pilaster y Cía. (Follett 2011, 64)

Este párrafo se refiere a hechos históricos. Sir Samuel Morton Peto (18091889) fue un destacado ingeniero y empresario victoriano, dedicado sobre todo a los ferrocarriles y a la construcción, incluyendo monumentos tan emblemáticos de Londres como la Plaza de Trafalgar y la Columna de Nelson. Quebró en 1866.

La Sociedad Bancaria de Londres también existió, aunque no desapareció entonces. La traducción correcta debería haber sido Sociedad Anónima Bancaria de Londres (en inglés la London Joint Stock Bank; Follett 1994, 47). En efecto,

\footnotetext{
${ }^{4}$ En inglés Senior Partner (Follett 1994, 42). La palabra partner, o socio, es más clara que presidente, y tiene importancia a la hora de asumir responsabilidades ante circunstancias adversas, como las que se van a producir en el futuro.
} 
hubo muchos debates a propósito de la autorización a las sociedades anónimas para dedicarse a la banca, que existía bajo la forma de socios propietarios, con un máximo de seis, y plenamente responsables con su patrimonio personal -en el Banco Pilaster eran cuatro. En 1826 se aprobó que los bancos pudieran ser sociedades anónimas siempre que no se radicaran a menos de 65 millas de Londres -son los "bancos provinciales" de los que hablaban los economistas clásicos. Siete años más tarde se levantó esta restricción, aunque los bancos que abrieran en Londres no podían emitir billetes, lo que protegía el monopolio del Banco de Inglaterra. Esta forma societaria finalmente se impuso, y desaparecieron los bancos que eran partnerships o sociedades personales o familiares, como sucedía con los Pilaster (Fetter 1965, 120-2, 165-7; Jenks 1963, 259-62; Kerridge 1992). ${ }^{5}$

Entre los primeros bancos que aprovecharon la nueva legislación más permisiva estaba el London Joint Stock Bank, fundado en 1836. Al revés de lo que sugiere Follett, no cerró el Black Friday de 1866; continuó sus actividades, se integró en 1917 en el Midland Bank y, muchos años después, en el HSBC.

El que sí desapareció en 1866 fue el banco Overend, Gurney \& Co., establecido en los años 1820 y que dominó el mercado londinense de descuento de letras en las tres décadas que siguieron (Collins 1992). Conviene recordar que, aunque desapareció, al final pagó todo lo que debía a sus acreedores, y el coste fue soportado en su totalidad por los accionistas, lo mismo que sucede en la novela con el Banco Pilaster (Hawtrey 1938, 83-5, 109).

Esta crisis desató la última carrera contra un banco que iba a producirse en Gran Bretaña hasta el descalabro de Northern Rock en el otoño de 2007 (Milne y Wood 2008, 517). La quiebra del Overend fue un elemento crucial en la redacción del primer tratado sobre la banca central moderna: Lombard Street, de Walter Bagehot -por cierto, la sede de Overend estaba en esa misma calle (Bignon, Flandreu y Ugolini 2012, 580; Clapham 1966, vol. 2, 261-3). ${ }^{6}$ A Bagehot, la bancarrota de Overend le parecía un "caso pasmoso" de mal gobierno del sistema crediticio, por la relevancia de la institución y sus impecables antecedentes: "La suspensión de pagos de Overend, Gurney \& Co. -la empresa privada que suscitaba la mayor confianza en Inglaterra- desató una alarma inédita por su sorpresa y magnitud" (Bagehot 1873, 18; véase también 183). Esta quiebra era para Bagehot la señal de que "no debemos confiar excesivamente en una buen imagen de larga data o en una tradición empresarial firmemente enraizada" (Bagehot 1873, 19), y se apoya en este caso para exponer su teoría sobre la

\footnotetext{
${ }^{5}$ Un reciente best-seller de Michael Lewis subraya el impacto en la burbuja especulativa y la crisis actual del cambio de las sociedades de valores norteamericanas, que pasaron de ser partnerships a ser sociedades por acciones (Lewis 2010, 263).

${ }^{6}$ Sobre el impacto de la crisis de Overend en la literatura popular de la época puede verse Michie 2001, 83.
} 
necesidad de un intervencionismo mayor del Banco de Inglaterra, que a su juicio tenía las reservas suficientes para frenar el pánico, evitando el contagio de una entidad a otras a lo largo del sistema, y no lo hizo. ${ }^{7}$ El caso ha sido comparado con la negativa de la Reserva Federal a rescatar a Lehman Brothers en septiembre de 2008 (Flandreau y Ugolini 2011, 4). La teoría del prestamista de última instancia se plasma con Bagehot pero tenía raíces anteriores, en particular el economista y banquero -y muy religioso, como los Pilaster- Henry Thornton (Milne y Wood 2008, 521; Laidler 1993, 36). ${ }^{8}$

¿Cuál era la actividad de Overend? Hemos visto que en la versión española de la novela de Follett se le llama "firma intermediaria de efectos mercantiles". La traducción parece correcta porque en inglés se habla de billbroker (Follett 1994, 46). Sin embargo, no es la actividad de corretaje la clave, porque los billbrokers crecieron, se asociaron, se capitalizaron y se convirtieron en discount houses, bancos de descuento que no sólo intermediaban sino que tomaban riesgo por su cuenta en forma de títulos, bonos o papeles comerciales diversos. Esto fue lo que sucedió con Overend, Gurney \& Co., que salió a bolsa en pleno auge en 1865. La burbuja estalló el año siguiente, los tipos de interés subieron del $3 \%$ al $7 \%$ entre julio de 1865 y enero de 1866, y, aunque se moderaron después, el Banco de Inglaterra se negó a rescatar a Overend, que fue declarada insolvente y cerró (Milne y Wood 2008, 522-523). ${ }^{9}$ Las inversiones de Overend habían sido malas, en particular en transportes y obras públicas -como ocurrirá

\footnotetext{
${ }^{7}$ Bagehot 1873, 191. Defiende un sistema bancario basado en sociedades anónimas, como el London Joint Stock Bank, que acabamos de mencionar: "Los bancos que son sociedades anónimas han tenido un notable éxito en nuestro país" y cree que esa forma societaria en la banca representa una excepción frente a las conocidas objeciones de Adam Smith contra las sociedades anónimas (Bagehot 1873, 243; Smith 2007, 696). En todo caso desconfía del partnership familiar, como el que tenían los Pilaster, y también el Overend: "Un negocio hereditario de enorme magnitud es peligroso. La gestión de una empresa de este tipo requiere más laboriosidad que la normal y más destreza que la normal. Y no hay ninguna seguridad de que dichas cualidades surjan con regularidad en cada generación. El caso de Overend, Gurney \& Co., el ejemplo característico de todo lo que está mal en el mundo de los negocios, es un caso particularmente alarmante de este peligro. Probablemente no se puedan encontrar hombres de negocios con más inteligencia (me refiero a inteligencia para los objetivos específicos de su profesión) que los fundadores y principales directivos de esta empresa. $\mathrm{Y}$ sin embargo, en unos pocos años, su gestión quedó en manos de una generación cuya insensatez superó los límites imaginables de la incapacidad. En un plazo muy breve convirtieron la prosperidad en ruina y la opulencia en insolvencia" (Bagehot 1873, 273). A pesar de todo, no pensaba que el intervencionismo era "natural" (Bagehot 1873, 67). Flandreau y Ugolini 2011 sugieren que el papel del Banco de Inglaterra en la crisis de Overend fue el de una suerte de prestamista de última instancia pero en un grado que no dio lugar a problemas de riesgo moral.

${ }^{8}$ En el momento de la crisis el Banco de Inglaterra subió el tipo de interés durante tres meses hasta el $10 \%$-el Banco de Francia, en contraste, lo mantuvo entre el 3,5 \% y el $4 \%$ (Patterson 1870, 226-228; véase el contraste crítico con Escocia en página 229; sobre Escocia véase también Hickson y Turner 2004, 914).

${ }^{9}$ Sobre el conflicto anterior entre Overend y el Banco de Inglaterra véase Clapham 1966, 242-8.
} 
con los Pilaster- y por tanto no era el suyo un mero problema de liquidez (Collins 1992; Flandreau y Ugolini 2011, 12). El proceso de decadencia de Overend fue el mismo que en el Banco Pilaster: cambió la gestión de la empresa, y sus nuevos responsables (en una etapa anterior, nótese, de reducción de los tipos de interés) abandonaron las estrategias prudentes de sus antecesores, embarcándose en inversiones cada vez más arriesgadas. El Economist comentó que Overend, Gurney \& Co. "se ha quedado con las peores letras de Inglaterra" (Mahate 1994, 103).

Overend arrastró a Tobías Pilaster. Su hijo Hugh, que desea reivindicar la figura de su padre -“fue a la bancarrota, pero nunca robó" (Follett 2011, 102) ${ }^{10}$ se revela un banquero diestro. No ha ido a la universidad, empezó temprano a trabajar y la banca es su única pasión profesional. De la mano de Hugh se explica el funcionamiento del Banco Pilaster, en particular el descuento de letras o la negociación de préstamos internacionales, concretamente para Rusia, cuya emisión de bonos al 5 con 3/8 \% de interés realiza el Banco Pilaster mediante suscripción, con lo que existe el riesgo de que no se puedan colocar en su totalidad y el banco deba comprar el excedente, un tema sobre el que se volverá a hablar al final del libro.

Se trata de un banco sólido en un país que crece. ${ }^{11}$ Circunstancias personales y conspiraciones varias, empero, hacen que Hugh deba marcharse a Boston en 1873. Lo hace llevando un puñado de libros entre los que destaca La riqueza de las naciones, y se añade que el joven Pilaster "consiguió aprender las tareas y procedimientos de trabajo de todos los departamentos con mucha mayor rapidez porque estudió previamente la teoría" (Follett 2011, 245). ${ }^{12}$ Su país de destino atravesaba agudas dificultades económicas: ${ }^{13}$

A principios del decenio de 1870, varios bancos habian concedido empréstitos importantes sobre la seguridad de valores especulativos ferroviarios, y cuando

${ }^{10}$ Para Adam Smith la distinción entre quiebra y quiebra fraudulenta eran tan trascendental que la pena de muerte en el segundo caso le parecía "sumamente justa". Consciente de que esto podía ser considerado excesivo, lo justifica por cuatro razones: el gran beneficio, la facilidad de ocultamiento del fraude, la gran pérdida para los acreedores, y la tentación u oportunidad del dolo (Smith 1982, 131-2).

${ }^{11}$ Se alude a la magnificencia de las casas de los ricos, y también en varias oportunidades al "impresionante" e "imponente" edificio del Banco Pilaster (Follett 2011, 237, 277); Hickson y Turner $(2004,907)$ subrayan la importancia de esas construcciones para la imagen de seguridad de los bancos, aunque la novela, que trata de un banco cuyo nombre significa "pilastra", no deja de apuntar que el banco de los Greenbourne es más sólido y de apariencia menos espectacular (Follett 2011, 383).

${ }^{12}$ Aparte de Robinson Crusoe, Ken Follett se permite la ironía de mencionar otros dos libros, sin duda inventados, Sistemas comerciales modernos y El empleado mercantil de éxito (Modern Business Methods y The Successful Commercial Clerk, Follett 1994, 206).

13 "La depresión de Estados Unidos de los años 1870 es famosa entre los economistas, porque es la más prolongada contracción cíclica de la historia del país" (Fels, 1951, 325). 
la construcción de líneas de ferrocarriles empezó a tener problemas, a mediados de 1873, tales bancos empezaron a dar la impresión de que se tambaleaban. Unos dias antes, Jay Cooke \& Co., agentes del gobierno estadounidense, fueron a la quiebra, y arrastraron consigo al First National Bank de Washington; y la noticia llegó a Londres el mismo día a través del cable transatlántico, telegráfico. Ahora, cinco bancos neoyorquinos, incluida la Union Trust Company -una entidad bancaria de suma importancia- y la Mechanic's Banking Association habian suspendido sus actividades. La Bolsa habia cerrado sus puertas. (Follett 2011, 245)

Todas esas entidades existieron y sus problemas hicieron eclosión en 1873 por una crisis de sobreinversión en los ferrocarriles (Sobel 1968, 154ss). Esto lo cuenta en la novela Danny Robinson, que tras el auge de dichas inversiones se queda sin empleo y decide volver a Inglaterra (Follett 2011, 258). ${ }^{14}$ La bolsa de Nueva York tomó la decisión sin precedentes de cerrar por un periodo indefinido -y tardó diez días en volver a abrir. La burbuja de los ferrocarriles, en un contexto de gran crecimiento de la producción y de caída de los precios mayoristas (Fels 1951, 330), ha sido comparada con las de finales del siglo XX y comienzos del siglo XXI: en su financiación los préstamos y los bonos ocuparon el lugar de las acciones (Mixon 2008, 722-5, 754). ${ }^{15} \mathrm{El}$ ambiente inversor se deterioró progresivamente, hasta el acontecimiento considerado más grave: la quiebra de Cooke (Mixon 2008, 726, 745; Studenski y Krooss 1952, 181). El propio Jay Cooke dio su versión de los hechos casi treinta años después: alegó que la financiación externa excesiva de los ferrocarriles se debió a los obstáculos de las propias autoridades a la venta de tierras públicas que ellas mismas habían cedido a las empresas ferroviarias, y añadió que la Guerra Francoprusiana frenó la llegada de capital extranjero (Cooke 1902, 582, 584). ${ }^{16}$

\section{LA CRISIS DE 1878}

La segunda parte del libro corresponde a 1879, y se abre con la ratificación de los talentos de Hugh Pilaster, que acaba de regresar de América. A sus 26 años es el primer ejecutivo del banco, porque ha hecho excelentes inversiones

\footnotetext{
${ }^{14}$ Danny da en esta página dos referencias históricas: trabajó en el "primer puente de acero" sobre el Mississippi, que se inauguraría en 1874, y en el ferrocarril Union Pacific, creado en la década anterior.

${ }^{15}$ El trabajo de Mixon, en contra de las tesis que habitualmente señalan la importancia del pánico como reflejo de reacciones más instintivas que razonadas, sostiene que hubo entonces respuestas racionales a unos riesgos percibidos correctamente (Mixon 2008, 723, 740).

${ }^{16}$ Menciona también la desmonetización de la plata de 1873, que le parece "una locura, si no un delito", y que no causó más daño por los ulteriores descubrimientos de oro en Estados Unidos y otros países (Cooke 1902, 586). Para un análisis de la peligrosa estrategia de Cooke de financiar inversiones a muy largo plazo véase Fels 1951, 336-40.
} 
en Estados Unidos, teniendo la habilidad de eludir los malos negocios, en particular los ferroviarios.

La malvada Augusta, que miente desde el principio, odia a los judíos y los homosexuales, y no sabe nada del mundo de la banca, urde una campaña para conseguir un título nobiliario para su marido, lo que consigue mediante la complicidad corrupta de la aristocracia, la política, y el periodismo, en éste último caso con Arnold Hobbes, personaje que se corrompe a cambio de puestos en consejos de Administración de algunas empresas, puestos que solían ocupar los merchank bankers (Beaumon 1992).

Otro banco en crisis entra en la trama de la novela. Dice Danny Robinson: "Por tercera vez en mi vida, unos banqueros incompetentes me han arruinado" (Follett 2011, 316). Su ruina es la de una institución: la Asociación para el Bienestar de los Trabajadores, mutualidad cuyos fondos fueron depositados en el Banco de la Ciudad de Glasgow. A raíz de esto se conversa sobre regulación e intervención de las autoridades en la banca, y otro personaje apunta:

Según he oído decir, es harto probable que los directores del Banco de la Ciudad de Glasgow vayan a la cárcel; y el gerente también. (Follett 2011, 321)

En 1879 nadie perdió su dinero y ya había banqueros presos. En la quiebra del City of Glasgow Bank, establecido en 1839, no hubo pánico, ni carreras. Se trató de un caso de fraude, que llevó a varios de sus propietarios y directivos a la cárcel, pero la solución fue bastante parecida a la que Hugh Pilaster contemplará años más tarde: otros bancos escoceses lo absorbieron, quedándose con sus activos y pasivos, y el banco desapareció (Milne y Wood 2008, 523). Llegó a estar más capitalizado que ningún otro banco de la ciudad, pero estaba expuesto al mercado monetario de Londres, y participaba activamente en las finanzas internacionales, descontando títulos de América y la India. Había tenido ya una sacudida en 1857, pero era considerado en el momento de su quiebra un banco sólido y su acción cotizaba al alza, aunque, como se supo después, debido a compras clandestinas de autocartera (Rosenblum 1933, 285). Tras una investigación que descubrió mucha concentración de préstamos impagados, el Banco de Escocia se negó a rescatarlo y el banco cerró en octubre de 1878 (Mahate 1994, 104-105).

Así como el caso Overend es considerado el causante del impulso a la política del prestamista de última instancia, el caso de City of Glasgow Bank es considerado la causa del cambio en la legislación bancaria británica, sobre la base de que la responsabilidad ilimitada en la banca era insostenible porque tendía a generar un problema de selección adversa: nadie rico querría ser banquero en tales circunstancias, que fomentarían la difusión de la propiedad de los bancos entre individuos con pocos recursos y por lo tanto poco que perder. La mayoría de los bancos británicos eran como el Banco Pilaster: propiedad de un grupo de socios acaudalados, con responsabilidad ilimitada y con un alto por- 
centaje de control de la empresa (el $100 \%$ en el caso de los Pilaster): se pensaba que el sistema así era estable, porque los propietarios responsables vetarían las transferencias de acciones para garantizar que todo propietario potencial tendría el dinero suficiente como para sostener el banco. Cuando el Banco de Glasgow cerró, los auditores independientes establecieron su desequilibrio patrimonial en más de 5 millones de libras. En el juicio, el gerente y uno de los consejeros fueron considerados culpables de falsear y adulterar el balance del banco, y fueron sentenciados a 18 meses de prisión; otros consejeros fueron hallados culpables de haber divulgado datos de esos balances que sabían que eran falsos, y por eso recibieron una sentencia de ocho meses de prisión. ${ }^{17}$ La quiebra, motivada entre otras razones por las inversiones en ferrocarriles, llevó a la bancarrota a unos 600 accionistas del banco, pero el déficit fue cubierto, y los depositantes -esto quizá refute lo que dice Danny Robinson- pudieron transferir sus fondos a otras entidades, salvo si eran accionistas. El desenlace legal fue la Companies Act de 1879, que, con la idea de prevenir la mencionada transferencia de acciones a personas sin recursos y asegurar la protección de los depositantes, estableció la responsabilidad limitada con una porción fija del capital como reserva no enajenable: la mayoría de los bancos se pasó al nuevo sistema (Acheson y Turner 2008, 236-240, 244, 251; Collins 1989, 505; Hawtrey 1938, 98-100). ${ }^{18}$

En este contexto de crisis, y a propósito de Córdoba, se habla de la "escasamente efectiva oposición liberal" del país (Follett 2011, 613). La expresión "liberal" se emplea en la novela en el sentido clásico, y se la relaciona con el mundo de los negocios. ${ }^{19}$ Las ideas del propio Ken Follett están más cercanas al socialismo moderado que al liberalismo. Amigo personal de Tony Blair, es desde hace muchos años un entusiasta del Partido Laborista, por el cual su segunda mujer, Barbara Follett, ocupó un escaño en el Parlamento en Londres. Esta novela es contraria al conservadurismo, y habla abiertamente de socialismo y reforma. Hugh es el gran héroe de los Pilaster, y no lo es por su conservadurismo sino por su bondad, honradez y esfuerzo (Ramet 1999, 113-117). En cuanto a la religión, aunque esta novela pondera a judíos y metodistas (mucho más a los primeros), puede apuntarse que Follett no es religioso (Ramet 1999, 112): firmó en 2010 un manifiesto oponiéndose a la visita del Papa Benedicto XVI al Reino Unido. Así como los ladrones de Under the streets of Nice eran de

${ }^{17}$ Hay detalles sobre el juicio en Rosenblum 1933.

${ }^{18}$ Hickson y Turner (2004) aducen en contra del free banking que para afrontar el problema de las asimetrías de información y la inconsistencia temporal resultaba mejor la responsabilidad personal ilimitada y era más estable, aunque no con limitación del número de socios.

${ }^{19}$ Recuérdese que Hugh parte hacia Estados Unidos con La riqueza de las naciones; y en su hora fatal, Seth Pilaster lee la publicación liberal por excelencia, que había aparecido en 1843 con el objetivo explícito de apoyar el libre comercio: The Economist (Follett 2011, 267; véase también 295, 301, 431). 
derechas (Maurice y Follett 1989), en Una fortuna peligrosa las heroínas que montan el hospital benéfico lo hacen en contra de los centros religiosos; los villanos del relato, encabezados por Augusta, tienen claro que dicho hospital "para madres solteras es un escándalo público", mientras que la heroína Maisie dice: "Aquí somos todas mujeres y comprendemos. No hablamos de maldad. Eso es para los curas y los políticos" (Follett 2011, 472, 515).

Aunque hay escasas alusiones en la novela a la economía y las finanzas de países concretos de América Latina, en esta parte hay una mención, con el objetivo claro de señalar los riesgos de invertir allí. En abril de 1879 un destacado empleado del banco le dice a Hugh Pilaster:

Yo trabajaba aquí cuando, hace quince años, el gobierno venezolano dejó al descubierto el pago de sus bonos. Mi padre, Dios lo tenga en su santa gloria, recordaba el incumplimiento de Argentina, en 1828. Y mire los bonos mexicanos... pagan dividendos de vez en cuando. (Follett 2011, 380)

La Argentina efectivamente suspendió pagos en 1828, y en esa década lo hicieron otras naciones latinoamericanas como Perú, Colombia (que entonces incluía Venezuela), Chile y México. A raíz de la crisis de comienzos de los años 1870 , volvieron a suspender pagos Perú, Colombia, Bolivia, Uruguay y varios países centroamericanos. La crisis siguiente, la de 1890, en la que entraremos a continuación, está especialmente asociada a la Argentina, que suspendió pagos, afectando a Baring; pero también lo hicieron otros países del subcontinente, incluyendo a Venezuela. No es extraña, pues, la actitud de Solly Greenbourne: "al igual que la mayoría de los inversores, desconfiaba de América del Sur" (Follett 2011, 443).

\section{LA CRISIS DE 1890}

La tercera y última parte de Una fortuna peligrosa transcurre en el año 1890. Ha muerto Joseph Pilaster, padre de Edward que sucedió a Seth en la presidencia del consejo del banco, y en la discusión sobre quién ha de ser nuevo Senior Partner aparece otra vez el contraste entre Augusta, que manipula con el argumento de que sólo cuenta la familia -"jtenemos derecho a elegir a quien nos parezca bien!"- y Hugh Pilaster, que replica: "No tenemos derechos, sólo deberes. Se nos han confiado millones de libras de otras personas. No podemos actuar como nos guste: tenemos que hacer lo que debemos hacer" (Follett 2011, 507-8).

Se explica la situación de los bonos de América del Sur: hasta ese momento han dado una elevada rentabilidad, y de ese negocio se ha ocupado Edward Pilaster, al que su madre Augusta pretende impulsar a la presidencia del consejo. Hugh anticipa el problema: los no fiables gobiernos sudamericanos puede dejar de honrar su deuda pública, con lo que el valor de sus bonos se hundiría; cuando Augusta le señala que él también vende bonos, aunque norte- 
americanos, contesta subrayando la estabilidad institucional de Estados Unidos. Finalmente, Hugh dimite como socio y hay una conversación con otro socio y hermano de su padre, el tío Samuel, en el que éste consigue que retrase su decisión aludiendo a los efectos de la misma, y Hugh acepta:

La crisis de una empresa podía provocar el hundimiento de otros negocios perfectamente sólidos, tal como la quiebra de Overend \& Gurney acabó con la firma del padre de Hugh en 1866. (Follett 2011, 512)

El Banco Pilaster se dispone a organizar un empréstito de gran cuantía para Córdoba: dos millones de libras, para construir un puerto en Santamaría -en realidad: para el golpe de Estado de los Miranda. Hugh explica que se estrecha el mercado sudamericano, con un dato que tiene importancia para lo que sucederá:

En cada una de las tres últimas emisiones el banco no ha tenido más remedio que comprar bonos en el mercado abierto para mantener su precio artificialmente. (Follett 2011, 552)

Logra el acuerdo de los socios a la propuesta de no garantizar la emisión, es decir, no suscribirla sino actuar sólo como comisionistas, con menos beneficio pero también menos riesgo. Se aprueba esta significativa variación por primera vez: siempre antes los bonos cordobeses habían sido suscritos por el banco. Sin embargo, Micky consigue que Edward realice un fraude y pase el contrato como si fuera uno de suscripción y no de corretaje. Cuando Hugh descubre la estafa, la emisión ya ha fracasado, sólo se han podido colocar cuatrocientas mil libras, pero los Miranda han retirado del banco un millón ochocientas mil, actuando como si la operación hubiera sido suscrita por el Pilaster. No obstante, Hugh precisa que el banco no está técnicamente en quiebra: han bajado las reservas pero "los bonos figuran en el activo de nuestro balance, valorados en su precio de compra. De modo que el pasivo queda compensado y somos solventes". Samuel Pilaster señala la condición crítica: el precio de esos bonos no puede caer, y Hugh ratifica que si se ese precio bajara "nos encontraríamos en graves dificultades" (Follett 2011, 571-2). La catástrofe podía evitarse: no tienen liquidez, pero tienen solvencia.

Finalmente, el acontecimiento más temido se produce: estalla la guerra en Córdoba, promovida por los Miranda. El país deja de pagar su deuda, y sus títulos se derrumban: ante esa caída abrupta del valor de su activo, el Banco Pilaster está quebrado.

La situación, la fecha y la exposición a América Latina evocan la crisis del Banco Baring, que fue aún más suave que la del Banco de Glasgow, lo que es habitualmente atribuido a que el Banco de Inglaterra actuó cada vez más como un banco central moderno, suspendiendo la convertibilidad, lo que significaba que el Banco de Inglaterra podía emitir mucho más allá de sus reservas de oro, 
aceptando como contrapartida títulos de deuda pública (Milne y Wood 2008, 523; Clapham 1966, vol. 2, 327-39).

Baring Brothers, fundado en el siglo XVIII, se especializó en los años 1880 en América Latina, durante una época de tipos de interés relativamente bajos, que no superaron el $4 \%$, cuando la rentabilidad de las inversiones latinoamericanas era superior. En Argentina tenía ocho veces más fondos prestados que su inmediato rival, y además tenía acciones de la compañía de aguas del país. Cuando en 1890 Argentina suspendió los pagos de los intereses, y la compañía de aguas quebró, el Gobierno británico era partidario de dejar caer a Baring, pero el Banco de Inglaterra se salió finalmente con la suya y orquestó un rescate, en el que también participaron bancos privados (Mahate 1994, 106-107). ${ }^{20}$ Baring, que había hecho con Argentina lo mismo que Pilaster tras la trampa de Edward, es decir, suscribir sus títulos, iba a durar todavía un siglo más, llegando a ser el banco de inversión británico más antiguo, hasta su colapso en 1995, a raíz de las operaciones ruinosas y fraudulentas en el mercado de derivados de su joven bróker en Singapur, Nick Leeson, que fue condenado a cuatro años de prisión en ese país.

A propósito de la banca, en este momento crucial de la novela habla por única vez el gobernador del Banco de Inglaterra. En la charla con Maisie y Danny Robinson se establece un paralelismo entre el hospital que dirige la primera y la legislación bancaria, a cuenta del riesgo moral y las externalidades negativas. Maisie le dice que el amparo a las madres solteras no padece ese riesgo porque no fomenta la inmoralidad: "Sólo les evitamos que den a luz en mitad de la calle", y Danny tercia:

- Es un poco como el proyecto de Ley Bancaria que presenté en el Parlamento, que obligaría a los bancos a suscribir un seguro a favor de los pequeños ahorradores o cuentacorrentistas.

- Lo sé- dijo el gobernador.

- No faltan críticos que aseguran que tal medida estimulará las quiebras, al hacerlas menos trágicas. Pero eso es una bobada. Ningún banquero quiere ir a la bancarrota, bajo ninguna circunstancia.

- Desde luego que no.

- Cuando un banquero realiza una operación no piensa que por culpa de su temeridad puede dejar sin un penique a una viuda de Bournemouth...sino que se preocupa de su propio capital. De modo análogo, el sufrimiento que puede producir engendrar un hijo ilegítimo no impide en absoluto a los hombres sin escrúpulos seducir a ingenuas sirvientas.

${ }^{20}$ Sayers, que subraya las abundantes inversiones realizadas por los británicos en el extranjero en los años anteriores, recuerda que los tipos de interés del Banco de Inglaterra bajaron en 1890, desde el $6 \%$ en enero hasta el $3 \%$ en mayo, a pesar de la crisis desatada por el impago de la Argentina (Sayers 1970, 5-6). 
- Comprendo su punto de vista -declaró el gobernador con expresión afligidaUn paralelismo de lo más... ah... original. (Follett 2011, 579-580)

Y eso es todo, lo que transmite la idea de que el Banco de Inglaterra no tenía un papel relevante en el mercado de bonos, pero esto, que podía ser cierto hasta mediados del siglo XIX, no lo era a finales, como acabamos de ver en el caso del banco Baring (King 1935, 321). El seguro de depósitos, por su parte, no fue oficialmente establecido hasta los años 1930.

Asimismo, al comienzo de esta tercera parte se menciona la intervención del Banco de Inglaterra en las crisis del Banco de Glasgow en 1878 y del banco francés Union Générale en $1882,{ }^{21}$ subiendo el tipo de interés "provisionalmente" hasta el $6 \%$, "muy por debajo del nivel de pánico" (Follett 2011, 496). ${ }^{22}$ El economista Ralph Hawtrey resumió así el éxito del banco emisor británico en la nueva emergencia de 1890: "Lo realmente notable de este caso es que no hubo crisis" (Hawtrey 1938, 105).

Sin Banco de Inglaterra, en la ficción, y sin un seguro que protegiese a los pequeños depositantes, en la realidad, Hugh, nombrado por fin presidente del consejo del Banco Pilaster, reúne a la familia para afrontar la situación. Lo que llama la atención del economista es que el banco quiebra y los que se quedan sin nada son los banqueros. La situación iba a ser muy diferente en el futuro, y hasta nuestros días se iban a suceder crisis bancarias más o menos profundas sin que los banqueros hayan perdido todos sus bienes. Esto último es lo que significa la responsabilidad ilimitada de los socios, y eso es lo que Hugh les dice a sus atribulados parientes: es $s u$ riqueza la que se ha esfumado junto con el banco.

Augusta se plantea la opción indecente:

Debemos salvar lo que podamos. Sin duda debe de haber todavía en el banco una buena cantidad de efectivo, oro y billetes de banco. Tenemos que sacarlo y esconderlo en algún lugar seguro antes de que se presenten los acreedores.

${ }^{21}$ Hawtrey 1938, 102. Este banco también existió, aunque tuvo una corta vida: había nacido en 1878. Inspiró a Zola su novela de 1891 El dinero.

${ }^{22}$ Collins, que apunta que el nivel de crisis es $7 \%$ o más señala, aparte del papel relevante del Banco de Inglaterra en las diversas crisis financieras, que en octubre de 1878 el tipo de interés subió del 5 al $6 \%$, pero bajó otra vez al $5 \%$ en noviembre, y en marzo de 1879 estaba en un 2,5 \% (Collins 1989, 523). Podemos aprovechar esta nota para apuntar que los datos que se dan sobre la evolución del bank rate en esta novela son en general correctos: en junio de 1873 subió al $7 \%$, en julio bajó al $6 \%$ y ese mismo mes al $3 \%$-en verdad llegó al 3,5\% el 31 de julio, y en agosto al $3 \%$-; en septiembre subió un punto hasta el $4 \%$. Un error destacado lo comete el hijo de Edward en septiembre de 1890 al decir que el tipo de interés estaba en el 2,5 $\%$, cuando en realidad estaba al doble de esa cifra; véase Hawtrey 1938, 284-9. Asimismo, se comenta la relación inversa entre el tipo de interés y las cotizaciones bursátiles (Follett 2011, $141,155,246,501,57)$. 
Pero Hugh la interrumpe:

No vamos a hacer semejante cosa. Ese dinero no es nuestro... Intenta meterte en la cabeza, tía Augusta, que no tienes ni una perra. ... Es la ley: cada uno de los socios ha de responder personalmente de las deudas del negocio. (Follett 2011, 586-7).

Esa dura realidad es difícil de aceptar, pero el mensaje es claro: toda esa familia de multimillonarios deberá vender sus casas, muebles, obras de arte, despedir a los sirvientes, y alquilar viviendas modestas en los suburbios. A partir de ese momento, la obsesión de Hugh no es reflotar el banco sino pagar las deudas, precisamente lo que en épocas posteriores resultó difuminado por la centrifugación de los riesgos hacia los contribuyentes a través de las autoridades monetarias y fiscales.

El grueso de la familia Pilaster sigue el consejo de Hugh y reduce su tren de vida y su bienestar con austeridad y honradez -algunos banqueros reales no lo hacían: el secretario del banco americano Union Trust Company, que mencionamos antes, se fugó con 250.000 dólares de la compañía (Mixon 2008, 726). De ahí el ahínco con el que Hugh persigue a Augusta, que pretende abandonar Inglaterra con las cajitas de rapé de su marido, que pueden valer cien mil libras: porque no le pertenecían a ella sino a los acreedores.

La venta de los bienes personales de los Pilaster para hacer frente a sus deudas, sin embargo, no será suficiente para reparar el daño causado, y de ahí que asistamos a una exposición sobre cómo resolver la inestabilidad y las posibles crisis de un sistema bancario sin respaldo oficial alguno. Eso es lo que Hugh le propone a Ben Greenbourne: un sindicato de bancos. No se trata de un rescate a la moderna usanza: no habrá dinero público de por medio, y ningún prestamista de última instancia rescatará al Banco Pilaster. ${ }^{23}$ Se trata de una inversión privada, aunque muy delicada, que Hugh presenta comparando el efecto que tendrá la crisis del Pilaster con el que tuvo la de Overend un cuarto de siglo antes:

Un montón de empresas se derrumbaron con aquella crisis. Pero lo de mañana será todavía peor. En los últimos veinticinco años, la cifra de negocio de la City se ha multiplicado por diez. Y al haberse hecho la banca tan compleja y aparatosa, las entidades bancarias estamos más interrelacionadas que nunca. Algunas personas cuyo dinero hemos perdido se encontrarán en la imposibilidad de liquidar sus deudas, de modo que también irán a la quiebra... y la cadena continuará. Dentro de ocho días, docenas de bancos se vendrán abajo, cientos de empresas se verán obligadas a echar el cierre y miles y miles de personas irán al

${ }^{23}$ Sobre el papel del prestamista de última instancia, el riesgo moral, y los problemas de la banca libre en esa época, véase Bignon, Flandreu y Ugolini 2012, 601. 
paro... a menos que emprendamos alguna acción para evitarlo...confio en que la comunidad bancaria haga algo. (Follett 2011,591)

Greenbourne se da cuenta del peligro de las externalidades negativas, pero al mismo tiempo rechaza que otros banqueros paguen las deudas del fallido Banco Pilaster. Se desarrolla a continuación un diálogo en el que Hugh empieza exponiendo su plan:

- Supongamos que se forma un sindicato de banqueros y que éste se hace cargo de los activos y pasivos del Pilaster. El sindicato garantizaría el pago de las deudas a todos los acreedores que lo solicitaran. Simultáneamente, el sindicato procedería a ir liquidando los activos del Pilaster de forma ordenada.

Greenbourne se sintió repentinamente interesado, y su irritación se volatilizó al considerar aquella original propuesta.

- Comprendo. Si los miembros del sindicato fueran lo bastante respetados y prestigiosos, su garantía quizá resultara suficiente para tranquilizar a todo el mundo y los acreedores no exigirían de inmediato su dinero. Con suerte, los ingresos producto de la venta de activos irían cubriendo los pagos a acreedores.

- Y se evitaría una crisis espantosa.

Greenbourne sacudió la cabeza.

- Pero, al final, los miembros del sindicato perderían dinero, porque las partidas de pasivo del Pilaster suman una cantidad mayor que las del activo.

- No necesariamente.

- ¿Cómo qué no?

- Disponemos de bonos de Córdoba por valor de más de dos millones de libras a los que hoy se les asigna valor cero. Sin embargo, nuestros otros activos son sustanciales. Todo depende en buena medida de la cantidad de dinero que podamos obtener mediante la venta de las casas y demás bienes de los socios; pero calculo que, actualmente, la diferencia en números rojos sólo es de un millón.

- Asi que el sindicato puede esperar perder un millón.

- Tal vez. Pero los bonos de Córdoba no van a carecer de valor eternamente. Es posible que los rebeldes sufran una derrota. O que el nuevo gobierno reasuma el pago de los intereses. En algún punto, la cotización de los bonos de Córdoba puede subir.... Con que los bonos lleguen a la mitad de su nivel anterior, el sindicato habrá recuperado su inversión. Y si suben más, el sindicato obtendría beneficio. (Follett 2011, 592-3)

Greenbourne parece entender la lógica de la operación, pero su cautela se refuerza al enterarse de que el éxito depende no sólo de que él participe en ella sino además que la encabece. Y entonces el argumento del riesgo moral se sobrepone al de las externalidades negativas, y el viejo banquero da marcha atrás: no hay que rescatar al imprudente. Al final, circunstancias personales hacen que Greenbourne cambie de opinión y respalde la operación, que se salda con éxito: los bonos cordobeses recuperan su valor y el sindicato de bancos obtiene un beneficio del $5 \%$. 
El Banco Pilaster desaparece, y con él la considerable fortuna de los socios. Hugh Pilaster se mantiene en el sector bancario, pero debe crear un banco nuevo, porque no puede resucitar el anterior; en cambio, sí puede continuar en el negocio porque conserva un capital importante: su propio crédito, su honradez personal y su talento empresarial y directivo. Comparte sus valores con la heroína, Maisie Robinson, con lo que cabe concluir que, como es habitual en la literatura popular, al final ganan los buenos, lo que no había sido siempre la norma de Follett. ${ }^{24}$ En este caso, además, sus aventuras se desenvuelven entre abundantes referencias financieras, en la mayoría de los casos correctamente expuestas y analizadas.

\section{CONCLUSIONES}

Una fortuna peligrosa tiene una característica destacable para economistas e historiadores: evoca con bastante fidelidad el contexto económico y financiero del siglo XIX, en particular en Inglaterra, aunque también se refiere a Estados Unidos. El único país inventado por Follett es Córdoba, aunque obviamente se trata de un país latinoamericano, y tampoco caben dudas sobre el motivo por el que el autor lo introduce en la novela: para resaltar el contraste entre los países con seguridad jurídica y los países donde esta seguridad no existe, por la aparición continua de guerras entre caudillos rivales y por la ausencia manifiesta de un marco institucional digno de tal nombre, lo que también tiene una evidente relevancia económica.

Las páginas de esta extensa novela recorren tres crisis que realmente tuvieron lugar: la del banco Overend, Gurney \& Co. en 1866, la del City of Glasgow Bank en 1878, y la mucho más conocida crisis de Baring de 1890. Se explica la operativa de los bancos y sus diferentes tipos de riesgo, en especial la financiación mediante préstamos y bonos de inversiones equivocadas a escala nacional e internacional, y la mala gestión. A pesar de que la novela se centra en un banco ficticio, el Pilaster, sus avatares reproducen lo que vivió la banca británica en el siglo XIX, un siglo de rápido crecimiento en la economía y las finanzas, y de grandes cambios regulatorios que van desde la Bank Charter Act de 1844 a la Companies Act de 1879, y al rescate de Baring por el Banco de Inglaterra. Esto cambios no vacunaron a la economía frente a las crisis, empero, como no lo harían los ulteriores, hasta hoy.

Las analogías entre el pasado que relata Ken Follett y el presente son claras, desde las burbujas especulativas financiadas con crédito bancario (entonces centrado en las obras públicas, pero a menudo de titularidad privada, como los

${ }^{24}$ En el prólogo que escribió para la edición de Paper Money que apareció en 1987 bajo su nombre verdadero, y cuando ya era un autor famoso, Follet dice: "hoy no estoy tan seguro como lo estaba en 1976 de los lazos entre el delito, las altas finanzas y el periodismo" (Follett 1987, 6). 
ferrocarriles), hasta las dificultades para organizar eficazmente la transición de la dirección en empresas familiares, pasando por la corrupción, los fraudes, y el tráfico de influencias en bancos, otras empresas, gobiernos y medios de comunicación.

Una diferencia con el presente, y con lo que sucedió realmente en 1890, cuando termina la novela, y de allí en adelante, resulta asimismo llamativa: el Banco Pilaster quiebra y es rescatado por un sindicato de bancos privados. La operación acaba con la enorme riqueza de los propietarios del banco, que asumen plena responsabilidad por todas las deudas impagadas, pero no cuesta nada a los contribuyentes, y la participación en ella de las autoridades, empezando por el Banco de Inglaterra, es nula.

\section{REFERENCIAS BIBLIOGRÁFICAS}

ACHESON, G.G. y TURNER, J.D. (2008). "The death blow to unlimited liability in Victorian Britain: The City of Glasgow failure" en Explorations in Economic History, 45 (3), pp. 235-253.

BAGEHOT, W. (1873). Lombard Street. A Description of the Money Market. Londres:

Henry S. King \& Co.

BEAUMAN, C. (1992). "Merchant banking". En Newman, P., Milgate, M. y Eatwell, J. (eds.): The New Palgrave Dictionary of Money \& Finance (pp. 697-700), Londres: Macmillan.

BIGNON, V., FLANDREAU, M., y UGOLINI, S. (2012). "Bagehot for beginners: the making of lender-of-last-resort operations in the mid-nineteenth century" en Economic History Review, 65 (2), pp. 580-608.

CLAPHAM, J.H. (1966). The Bank of England. Cambridge: Cambridge University Press.

COLLINS, M. (1989). "The banking crisis of 1878" en Economic History Review, 42 (4), pp. 504-527.

COLLINS, M. (1992). "Overend Gurney crisis". En Newman, P., Milgate, M. y Eatwell, J. (eds.): The New Palgrave Dictionary of Money \& Finance (p. 101), Londres: Macmillan.

COOKE, J. (1902). "A Decade of American Finance" en North American Review, 175 (noviembre), pp. 577-586.

FELS, R. (1951). "American business cycles, 1865-79" en American Economic Review, 41 (3), pp. 325-349.

FETTER, F.W. (1965). Development of British monetary orthodoxy, 1797-1875. Cambridge: Cambridge University Press.

FLANDREAU, M., y UGOLINI, S. (2011). "Where it all began: lending of last resort and the Bank of England during the Overend, Gurney Panic of 1866" en EHES Working Papers in Economic History, European Historical Economics Society, 7 (febrero), pp. $1-46$. 
FOLLETT, K. (1987). Paper money. Nueva York: William Morrow and Company, Inc.

FOLLETT, K. (1994). A Dangerous Fortune. Londres: Pan Books Macmillan.

FOLLETT, K. (2011). Una fortuna peligrosa. Traducción de Manuel Bartolomé, $6^{a}$ edición, Barcelona: Random House Mondadori.

FOLLETT, K. (s.d.). Página web, http://ken-follett.com/bibliography/a_dan gerous_fortune/, último acceso 1 diciembre 2012.

HAWTREY, R.G. (1938). A Century of Bank Rate. Londres: Longmans.

HICKSON, C.R. y TURNER, J.D. (2004). "Free banking and the stability of early jointstock banking" en Cambridge Journal of Economics, 28 (6), pp. 903-919.

JENKS, L.H. (1963). The migration of British capital to 1875. Londres: Nelson.

KERRIDGE, K. (1992). “Joint stock banks”. En Newman, P., Milgate, M. y Eatwell, J. (eds.): The New Palgrave Dictionary of Money \& Finance (pp. 552-553), Londres: Macmillan.

KING, W.T.C. (1935). "The Extent of the London Discount Market in the Middle of the Nineteenth Century" en Economica, N.S., 2 (7), pp. 321-326.

LAIDLER, D. (1993). The Golden Age of the Quantity Theory. The Development of Neoclassical Monetary Economics 1870-1914. Londres: Harvester Wheat-sheaf.

LEWIS, M. (2010). The Big Short. Londres: Allen Lane.

LINDE, L.M. (2004). "El banquero no bromea” en Revista de Libros, 85, enero, pp. 3338.

MAHATE, A.A. (1994). "Contagion Effects of Three Late Nineteenth Century British Bank Failures" en Business and Economic History, 23 (1), pp 102-115.

MAURICE, R.L. y FOLLETT, K. (1989). Under the streets of Nice. Nueva York: PaperJacks.

MICHIE, E. B. (2001). "Buying brains: Trollope, Oliphant, and vulgar Victorian commerce" en Victorian Studies, 44 (1), pp. 77-97.

MILNE, A. y WOOD,, G. (2008). "Banking Crisis Solutions Old and New", en Federal Reserve Bank of St. Louis Review, 90 (5), pp. 517-530.

MIXON, S. (2008). "The Crisis of 1873: Perspectives from Multiples Asset Classes" en Journal of Economic History, 68 (3), pp. 722-757.

PATTERSON, R. H (1870). "On our home monetary drains, and the crisis of 1866 " en Journal of the Statistical Society of London, 33 (2), pp. 216-242.

PERDICES DE BLAS, L. y SANTOS REDONDO, M. (coords.) (2006). Economía y Literatura. Madrid: Ecobook.

RAMET, C. (1999). Ken Follett: the transformation of a writer. Bowling Green, Ohio: Bowling Green State University Popular Press.

RAMET, C. (2010). "Ken Follett and the Scribbler's Trade: A Midnight Train to Somewhere" en Studies in Popular Culture; 33 (1), pp. 79-93.

ROSENBLUM, L. (1933). "The failure of the City of Glasgow Bank" en Accounting Review, 8 (4), pp. 285-291.

SAYERS, R.S. (1970). Bank of England operations, 1890-1914. Westport Conn.: Greenwood Press.

SMITH, A. (1981), An Inquiry Into the Nature and Causes of the Wealth of Nations. Campbell, R. H. y Skinner, A. S. (eds.), Indianápolis: Liberty Fund. 
SMITH, A. (1982). Lectures On Jurisprudence. Meek, R. L., Raphael, D. D. y Stein, P. G. (eds.), Indianápolis: Liberty Fund.

SMITH, A. (2007). La riqueza de las naciones. Madrid: Alianza.

SOBEL, R. (1968). Panic on Wall Street: a History of America's Financial Disasters. Nueva York: Macmillan.

STUDENSKI, P. y KROOSS, H.E. (1952). Financial history of the United States: fiscal, monetary, banking and tariff, including financial administration and local finance. Nueva York: McGraw-Hill. 
